\title{
GROUP ALGEBRAS AND ALGEBRAS OF GOLOD-SHAFAREVICH
}

\author{
PLAMEN N. SIDEROV
}

\begin{abstract}
In [2], Golod, using results of Golod and Shafarevich [1], has constructed a finitely generated algebra $A=K\left\langle y_{1}, \ldots, y_{d}\right\rangle$, over any field $K$, such that the ideal generated by $y_{1}, \ldots, y_{d}$ is nil, but $\operatorname{dim}_{K} A=\infty$. Moreover, when char $K=p>0$, the subgroup $G$ of the group of units of $A$, generated by $1+y_{1}, \ldots, 1+y_{d}$, is an infinite $p$-group. The main purpose of the present paper is to show that $K[G]$, the group algebra of $G$ over $K$, is not isomorphic to $A$ for "most" Golod-Shafarevich groups $G$.
\end{abstract}

In the celebrated paper [2] (cf. also [3]) Golod, using results of Golod and Shafarevich [1], constructed a finitely generated algebra $A=K\left\langle y_{1}, \ldots, y_{d}\right\rangle$ over any field $K$, such that the ideal (= two-sided ideal) generated by $y_{1}, \ldots, y_{d}$ is nil, but $\operatorname{dim}_{K} A=\infty$. This solves a problem of Kurosh [4]. Moreover, when char $K=p>$ 0 , the subgroup $G$ of the group of units of $A$, generated by $1+y_{1}, \ldots, 1+y_{d}$, is an infinite $p$-group, which solves a problem of Burnside.

The group $G$ appears to be a natural candidate for testing the validity of another finiteness problem, due to Kaplansky [5]: If $H$ is a finitely-generated group, such that the augmentation ideal of the group algebra $K[H]$ is nil, is it true that $H$ is finite? Indeed, it is known that the hypothesis implies that char $K=p>0$ and $H$ is a $p$-group, so that the existence of a $K$-algebra isomorphism $K[G] \cong A$ would solve Kaplansky's problem in the negative. The question whether such an isomorphism exists was posed to the author by A. L. Shmel'kin. The main purpose of the present paper is to show that for "most" Golod-Shafarevich groups $G, K[G]$ is not isomorphic to $A$.

In order to give precise statements, we need some notation. In what follows, $K$ denotes a fixed field of characteristic $p>0$, and $F=K\left\langle x_{1}, \ldots, x_{d}\right\rangle$ is the free associative algebra on the $x_{i}$ 's $(d \geq 2)$; we write $F^{+}$for its ideal, consisting of all polynomials without constant term. An ideal $I \subset F^{+}$is called a Golod-Shafarevich ideal if it has the following properties:

(1) $I$ is homogeneous;

(2) $\operatorname{dim}_{K}(F / I)=\infty$;

(3) every $(d-1)$-generated subalgebra of $A^{+}=F^{+} / 1$ is nilpotent.

THEOREM. Let I be a Golod-Shafarevich ideal, which is generated by homogeneous polynomials $f_{1}, f_{2}, \ldots$ such that $f_{1}=\left(x_{1}-x_{2}\right)^{p^{n}}$ and $\operatorname{deg} f_{i}>p^{n}+1$ $(i \geq 2)$ for some natural number $n$. Then the $K$-algebras $k[G]$ and $A=F / I$ are not isomorphic.

Received by the editors May 9, 1986.

1980 Mathematics Subject Classification (1985 Revision). Primary 16A22, 16A27; Secondary $20 \mathrm{~F} 50$. 
To see that such an $I$ does exist, recall from [3] that if $I$ is a homogeneous ideal, generated by $r_{i}$ elements in degree $i$, then (2) above holds provided there exists a real positive $\varepsilon>0$ such that

$$
r_{i} \leq \varepsilon^{2}(d-2 \varepsilon)^{i-2} .
$$

Furthermore, the proof of the theorem in [3] shows that if $f_{1}, \ldots, f_{j}$ are fixed and satisfy $(*)$, then one can find $f_{j+1}, f_{j+2}, \ldots$, which satisfy $(*)$, are of degree higher than $\max \left(\operatorname{deg} f_{1}, \ldots, \operatorname{deg} f_{j}\right)$, and such that $I$ has (3).

The Theorem raises the question of measuring the "difference" between $K[G]$ and $A$. We only note here that this cannot be done in terms of the growth of the algebras [6]. In fact, if $A$ satisfies condition (*), it grows exponentially (this follows from the proof of Lemma 2 in [2]). Now the canonical epimorphism $K[G] \rightarrow A$ gives an exponential lower bound on the growth of $K[G]$, while an exponential upper bound follows from its finite generation. (I am grateful to D. Anick for drawing my attention to the exponential growth of $A$ and for providing me with a proof that, with a single exception, $\operatorname{dim}_{K}(A)=\infty$ already implies exponential growth.)

Finally, we note these remarks imply that Golod-Shafarevich groups always grow exponentially which shows they are markedly different from the Grigorchuk counterexamples to the Burnside problem, which can have intermediate growth [7].

We shall use the following well-known lemma:

LEMMA 1. Let $a=\sum_{i \geq 0} a_{i} p^{i}, b=\sum_{i \geq 0} b_{i} p^{i}$ be the $p$-adic expansions of the natural numbers $a$ and $b$. Then

$$
\left(\begin{array}{l}
a \\
b
\end{array}\right) \equiv \prod_{i \geq 0}\left(\begin{array}{l}
a_{i} \\
b_{i}
\end{array}\right) \quad(\bmod p) .
$$

ProOF. Consider the polynomial $(1+x)^{a}$ over the field $G F(p)$ with $p$ elements. One has

$$
(1+x)^{a}=\prod_{i \geq 0}(1+x)^{a_{i} p^{i}}=\prod_{i \geq 0}\left(1+x^{p^{i}}\right)^{a_{i}} .
$$

The claim follows by equating the coefficients of $x^{b}$.

LEMMA 2. Let $a$ and $n$ be natural numbers. Then

$$
(1+a)\left(\begin{array}{c}
a \\
p^{n}-1
\end{array}\right) \equiv 0 \quad(\bmod p)
$$

Proof. One has

$$
p^{n}-1=(p-1) p^{0}+(p-1) p+\cdots+(p-1) p^{n-1} .
$$

Let $a=a_{0} p^{0}+a_{1} p+\cdots+a_{n-1} p^{n-1}+a_{n} p^{n}+\cdots$ with $0 \leq a_{i} \leq p-1$. Assume $p$ does not divide $\left(\begin{array}{c}a \\ p^{n}-1\end{array}\right)$. Then by Lemma $1 p$ does not divide $\left(\begin{array}{c}a_{i} \\ p-1\end{array}\right)$ for $i=0,1, \ldots, n-1$. Since $a_{i} \leq p-1$, one necessarily has $a_{i}=p-1$ for these $i$, hence

$$
a=p^{n}-1+a_{n} p^{n}+\cdots,
$$

which gives $1+a \equiv 0(\bmod p)$.

For a word $g=g\left(x_{1}, \ldots, x_{d}\right)$ in $x_{1}, \ldots, x_{d}$ we denote by $g_{i}$ the homogeneous polynomial of degree $i$ in the expansion of $g\left(1+x_{1}, \ldots, 1+x_{d}\right)$. Clearly, $g_{i} \in$ $G F(p)\left\langle x_{1}, \ldots, x_{d}\right\rangle$. 
LEMMA 3. For $g$ as above, assume $\operatorname{deg}_{x_{1}} g \equiv \operatorname{deg}_{x_{2}} g \equiv 0(\bmod p)$. Then the monomials $x_{1}^{p^{n}-1} x_{2}$ and $x_{1}^{p^{n}-1} x_{2} x_{1}$ have the same coefficient in the expansions of $g_{p^{n}}$ and $g_{p^{n}+1}$ respectively.

PROOF. Set $\operatorname{deg}_{x_{1}} g=p t, \operatorname{deg}_{x_{2}} g=p u$. We shall argue by induction on $u$. The claim is obvious for $u=0$. Assuming the claim for $u=s$, we shall prove it when $\operatorname{deg}_{x_{2}} g=p s+p$. Fixing $p$ arbitrary entries of $x_{2}$ in $g\left(x_{1}, \ldots, x_{d}\right)$, we write

$$
g\left(x_{1}, \ldots, x_{d}\right)=g_{1}\left(x_{1}, \ldots, x_{d}\right) x_{2} g_{2}\left(x_{1}, \ldots x_{d}\right) x_{2} \cdots x_{2} g_{p+1}\left(x_{1}, \ldots, x_{d}\right)
$$

and set

$$
h\left(x_{1}, \ldots, x_{d}\right)=g_{1}\left(x_{1}, \ldots, x_{d}\right) g_{2}\left(x_{1}, \ldots, x_{d}\right) \cdots g_{p+1}\left(x_{1}, \ldots, x_{d}\right) .
$$

By the induction hypothesis, the statement of the lemma holds for $h\left(1+x_{1}, \ldots, 1+x_{d}\right)$. Denote by $m(0 \leq m<p)$ the common coefficient of $x_{1}^{p^{n}-1} x_{2}$ and $x_{1}^{p^{n}-1} x_{2} x_{1}$. Writing $\operatorname{deg}_{x_{1}} g_{i}=t_{i}(i=1,2, \ldots, p+1)$, one has $t_{1}+t_{2}+\cdots+$ $t_{p+1} \equiv 0 \quad(\bmod p)$. Besides the occurrences of $x_{1}^{p^{n}-1} x_{2}$ and $x_{1}^{p^{n}-1} x_{2} x_{1}$, which come from $h\left(1+x_{1}, \ldots, 1+x_{d}\right)$, these monomials will appear in the expansion of $g\left(1+x_{1}, \ldots, 1+x_{d}\right)$ only in products which involve the letter $x_{2}$ from one of the fixed positions. It is easily seen that $x_{1}^{p^{n}-1} x_{2}$ has $C$ such occurrences, where

$$
C=\left(\begin{array}{c}
t_{1} \\
p^{n}-1
\end{array}\right)+\left(\begin{array}{c}
t_{1}+t_{2} \\
p^{n}-1
\end{array}\right)+\cdots+\left(\begin{array}{c}
t_{1}+t_{2}+\cdots+t_{p} \\
p^{n}-1
\end{array}\right)
$$

while the number of new occurrences of $x_{1}^{p^{n}-1} x_{2} x_{1}$ is

$$
\begin{aligned}
B= & \left(\begin{array}{c}
t_{1} \\
p^{n}-1
\end{array}\right)\left(t_{2}+t_{3}+\cdots+t_{p+1}\right)+\left(\begin{array}{c}
t_{1}+t_{2} \\
p^{n}-1
\end{array}\right)\left(t_{3}+\cdots+t_{p+1}\right) \\
& +\cdots+\left(\begin{array}{c}
t_{1}+\cdots+t_{p} \\
p^{n}-1
\end{array}\right) t_{p+1} .
\end{aligned}
$$

We shall prove that for each $i=1,2, \ldots, p$ one has

$$
\left(\begin{array}{c}
t_{1}+\cdots+t_{i} \\
p^{n}-1
\end{array}\right) \equiv\left(\begin{array}{c}
t_{1}+\cdots+t_{i} \\
p^{n}-1
\end{array}\right)\left(t_{i+1}+\cdots+t_{p+1}\right) \quad(\bmod p)
$$

Indeed, noting that $t_{1}+\cdots+t_{p+1}=p t$, and setting $a=t_{1}+\cdots+t_{i}$, Lemma 2 yields

$$
\left(\begin{array}{c}
a \\
p^{n}-1
\end{array}\right) \equiv\left(\begin{array}{c}
a \\
p^{n}-1
\end{array}\right)(-a) \equiv\left(\begin{array}{c}
a \\
p^{n}-1
\end{array}\right)(p t-a) \quad(\bmod p) .
$$

Then $C \equiv B(\bmod p)$, hence $m+C=m+B$, which proves the lemma.

We again use the notation introduced before Lemma 3:

LEMMA 4. Let $n$ be a natural number. There is no word $g$ with the following properties:

(1) $x_{1}^{i}, x_{2}^{i}$ do not enter in the expansion of $g_{i}$ for $i=1,2, \ldots, p^{n}-1$,

(2) $g_{p^{n}}=\left(x_{1}-x_{2}\right)^{p^{n}}$

(3) $g_{p^{n}+1}$ is in the ideal on $K\left\langle x_{1}, \ldots, x_{d}\right\rangle$ generated by $\left(x_{1}-x_{2}\right)^{p^{n}}$.

PROOF. Assume such a $g$ exists, with $\operatorname{deg}_{x_{1}} g=t, \operatorname{deg}_{x_{2}} g=s$. The monomial $x_{1}^{k}$ occurs in the expansion of $\tilde{g}=g\left(1+x_{1}, \ldots, 1+x_{d}\right)$ with coefficient $\left(\begin{array}{l}t \\ k\end{array}\right)$. Write 
$t=t_{0} p^{0}+t_{1} p+\cdots+t_{n} p^{n}+\cdots$ with $0 \leq t_{i} \leq p-1$. From (1) and Lemma 1 we have that $t_{i}=0$ for $i=0,1, \ldots, n-1$, so that $t=p^{n} t^{\prime}\left(t^{\prime} \in N\right)$. Similarly one obtains $s=p^{n} s^{\prime}$.

All homogeneous polynomials of degree $p^{n}+1$ which lie in the ideal generated by $\left(x_{1}-x_{2}\right)^{p^{n}}$ are $K$-linear combinations of the polynimials $u_{i}=\left(x_{1}-x_{2}\right)^{p^{n}} x_{i}$ and $v_{i}=x_{i}\left(x_{1}-x_{2}\right)^{p^{n}}(i=1,2, \ldots, d)$. From (3) one has

$$
g_{p^{n}+1}=\sum \alpha_{i} u_{i}+\sum \beta_{i} v_{i}, \quad \alpha_{i}, \beta_{i} \in K
$$

Since, by Lemma $1, x_{1}^{p^{n}+1}$ and $x_{2}^{p^{n}+1}$ do not occur in $g_{p^{n}+1}$, one has $\alpha_{1}=-\beta_{1}$ and $\alpha_{2}=-\beta_{2}$. Thus

$$
g_{p^{n}+1}=\alpha_{1}\left[\left(x_{1}-x_{2}\right)^{p^{n}}, x_{1}\right]+\alpha_{2}\left[\left(x_{1}-x_{2}\right)^{p^{n}}, x_{2}\right]+\sum_{i \geq 3} \alpha_{i} u_{i}+\sum_{i \geq 3} \beta_{i} v_{i}
$$

(square brackets denote commutators).

Since $\left[\left(x_{1}-x_{2}\right)^{p^{n}},\left(x_{1}-x_{2}\right)\right]=0$, i.e. $\left[\left(x_{1}-x_{2}\right)^{p^{n}}, x_{1}\right]=\left[\left(x_{1}-x_{2}\right)^{p^{n}}, x_{2}\right]$, one gets finally

$$
g_{p^{n}+1}=\left(\alpha_{1}+\alpha_{2}\right)\left[\left(x_{1}-x_{2}\right)^{p^{n}}, x_{2}\right]+\sum_{i \geq 3} \alpha_{i} u_{i}+\sum_{i \geq 3} \beta_{i} v_{i}
$$

It is easily seen that this polynomial contains no term $x_{1}^{p^{n}-1} x_{2} x_{1}$. By Lemma 3 , $x_{1}^{p^{n}-1} x_{2}$ cannot occur in $g_{p^{n}}$, which contradicts (2).

Let $I$ be an ideal in $F^{+}$. Denote by $J_{I}$ the ideal generated by all polynomials in $I$ of the form $1-g\left(1+x_{1}, \ldots, 1+x_{d}\right)$, where $g\left(x_{1}, \ldots, x_{d}\right)$ ranges over all words.

LEMMA 5. If $I$ is the ideal of the Theorem, then $J_{I} \varsubsetneqq I$.

ProOF. The claim is obvious if $J_{I}$ is not homogeneous. Assume it is. We shall prove $f_{1}=\left(x_{1}-x_{2}\right)^{p^{n}} \notin J_{I}$. Assuming $f_{1} \in J_{I}$, let

$$
f_{1}=\sum\left(1-u_{i}\left(1+x_{1}, \ldots, 1+x_{d}\right)\right)+\sum h_{1 j}\left(1-g_{j}\left(1+x_{1}, \ldots, 1+x_{d}\right)\right) h_{2 j},
$$

where $u_{i}\left(x_{1}, \ldots, x_{d}\right)$ and $g_{j}\left(x_{1}, \ldots, x_{d}\right)$ are monomials, $1-u_{i}\left(1+x_{1}, \ldots, 1+x_{d}\right) \in I$, $1-g_{j}\left(1+x_{1}, \ldots, 1+x_{d}\right) \in I$, and $h_{1 j}$ or $h_{2 j} \neq 1$. Since $I$ contains no homogeneous elements of degree smaller than $p^{n}$, the minimal degree of all monomials from the second sum is strictly higher than $p^{n}$. Therefore, there is is the first sum a summand, say $u=1-u_{1}\left(1+x_{1}, \ldots, 1+x_{d}\right)$, of a minimal degree $p^{n}$. Let $u=h_{1}+h_{2}+\cdots+h_{p^{n}}+h_{p^{n}+1}+\cdots$ with $h_{i}$ a homogeneous polynomial of degree $i$. Since $J_{I}$ is homogeneous, one necessarily has $h_{i}=0$ for $i=1,2, \ldots, p^{n}-1$ and $h_{p^{n}}=\left(x_{1}-x_{2}\right)^{p^{n}}$. Also, the hypotheses of the lemma imply $h_{p^{n}+1}$ is in the ideal generated by $\left(x_{1}-x_{2}\right)^{p^{n}}$. However, this is a contradiction by Lemma 4 .

For an ideal $I \subset F^{+}$, let $y_{i}$ denote the image of $x_{i}$ under the natural map $\varphi: F \rightarrow A=F / I$ and let $z_{i}$ be its image under the natural map $\psi: F \rightarrow B=F / J_{I}$. Assuming $y_{i}$ is nilpotent in $A$, it is easily seen that $z_{i}$ is nilpotent in $B$. Denote by $G$ the subgroup of (the group of units of) $A$, generated by the elements $1+y_{i}$, and by $H$ the subgroup of $B$, generated by $1+z_{i}(i=1, \ldots, d)$.

LEMMA 6. The groups $G$ and $H$ are isomorphic.

PROOF. The restriction on $H$ of the canonical epimorphism $\eta: B \rightarrow A$ maps $z_{i}$ to $y_{i}$, hence defines a surjection $H \rightarrow G$. Let $g=g\left(1+z_{1}, \ldots, 1+z_{d}\right)$ be in Ker $\eta$. 
Then $1=\eta(g)=g\left(1+y_{1}, \ldots, 1+y_{d}\right)$ means that $1-g\left(1+x_{1}, \ldots, 1+x_{d}\right)$ is in I. According to the definition of $J_{I}$, one has $1-g\left(1+x_{1}, \ldots, 1+x_{d}\right) \in J_{I}$, i.e. $g\left(1+z_{1}, \ldots, 1+z_{d}\right)=1$. The lemma is proved.

PROOF OF THE THEOREM. Let $T$ be a group generated by $t_{1}, \ldots, t_{d}$ and isomorphic to the group $G$ of the Theorem. Since the algebra $B$ (with 1 ) is generated over $K$ by the group $H$ (in the notation introduced before Lemma 6), the map $t_{i} \rightarrow 1+z_{i}$ extends to a surjective ring homomorphism $\rho: K[T] \rightarrow B$. We denote by $\pi$ the composition of $\rho$ and $\eta$.

This is an epimorphism with $\pi\left(t_{i}\right)=1+y_{i}$, and it maps the augmentation ideal $\omega$ of $K[T]$ onto $A^{+}$. Thus for every natural $k, \pi\left(\omega^{k}\right)=\left(A^{+}\right)^{k}$, so that one has a surjection $\pi_{k}: K[T] / \omega^{k} \rightarrow A /\left(A^{+}\right)^{k}$. By Lemma $5,0 \neq \operatorname{Ker} \eta$, so Ker $\pi \neq 0$. Choose $0 \neq f \in \operatorname{Ker} \pi$. Since the Golod-Shafarevich groups are residually finite (cf. [2, last line]), one concludes from a result of $\mathrm{Mal}^{\prime}$ tsev [8] that $\bigcap_{i=1}^{\infty} \omega^{i}=0$. Thus, there is a $k$ such that $f \notin \omega^{k}$, hence $\operatorname{Ker} \pi_{k} \neq 0$.

Assume there exists an isomorphism $\sigma: K[T] \rightarrow A$. For any $t \in T, 1-t$ is nilpotent, hence $\sigma(1-t) \in A^{+}$, which shows $\sigma(\omega) \subseteq A^{+}$. The reverse inclusion is clear. It follows that $\sigma$ induces an isomorphism $K[T] / \omega^{k} \cong A /\left(A^{+}\right)^{k}$ for any $k$. In particular these vector spaces have the same (finite) dimension. This contradicts Ker $\pi_{k} \neq 0$, and completes the proof of the Theorem.

I should like to express my thanks to L. L. Avramov for many helpful discussions concerning this problem.

\section{REFERENCES}

1. E. S. Golod and I. R. Shafarevich, On the class field tower, Izv. Akad. Nauk. SSSR Ser. Mat. 28 (1964), 261-272. (Russian)

2. E. S. Golod, On nil-algebras and finitely approximable p-groups, Izv. Akad. Nauk SSSR Ser. Mat. 28 (1964), 273-276. (Russian)

3. __, On some problems of Burnside type, Proc. Internat. Congr. Math., Moscow, 1966, pp. 284-289.

4. A. G. Kurosh, Problems in the theory of rings, related to Burnside problem on periodic groups, Izv. Akad. Nauk SSSR Ser. Mat. 5 (1941), 233-241.

5. I. Kaplansky, Problems in the theory of rings, Report of Conference of Linear Algebras, NASNRC Publ. 502, Washington, D.C., 1957, pp. 1-3.

6. W. Borho and H. Kraff, Über die Gelfand-Kirilov Dimension, Math. Ann. 220 (1976), 1-24.

7. R. I. Grigorchuk, Degrees of growth of finitely generated groups, and the theory of invariant means, Izv. Akad. Nauk SSSR Ser. Mat. 48 (1984), 939-985. (Russian)

8. A. I. Mal'tsev, Generalized nilpotent algebras and their associated groups, Mat. Sb. 25 (1949), 347-366.

Mathematics Institute, Bulgarian ACAdemy of Sciences, P.O. BoX 373, 1090 SOFIA, BULGARIA 